\title{
Ahmed valve implantation in childhood glaucoma associated with Sturge-Weber syndrome: our experience
}

\author{
Jaya Kaushik ${ }^{1,2} \cdot$ Jitendra Kumar Singh Parihar $^{1} \cdot$ Vaibhav Kumar Jain $^{3} \cdot$ Vijay Mathur $^{1}$
}

Received: 7 June 2018 / Revised: 8 August 2018 / Accepted: 13 August 2018 / Published online: 18 October 2018

(c) The Royal College of Ophthalmologists 2018

\begin{abstract}
Purpose To evaluate the efficacy and safety of Ahmed valve implantation in childhood glaucoma associated with SturgeWeber syndrome (SWS).

Methods A retrospective chart review of patients who underwent primary Ahmed valve implantation in childhood glaucoma associated with SWS. The outcome measures were success rate (Intraocular pressure (IOP) $>5$ and $<21 \mathrm{~mm} \mathrm{Hg}$ without additional surgical maneuver), and operative complications.

Results Twenty-four eyes of 18 patients were analyzed. Mean IOP $(26.21 \pm 3.52$ to $12.27 \pm 1.27 \mathrm{~mm} \mathrm{Hg})$ and mean number of anti-glaucoma medicines $(3.92 \pm 0.28$ to $1.5 \pm 1)$ reduced significantly after the surgery at the mean follow-up of $2.12 \pm$ 0.87 years $(P=<0.05)$. The cumulative probability of success rate was $75 \%$ (Confidence Interval $95 \%, 60$ to $100 \%$ ) by Kaplan-Meier survival curve analysis. Failed eyes [6 (25\%)], hyphema [4 (16.67\%)], hypotony [3 (12.5\%)], choroidal detachment [3 (12.5\%)], tube-cornea touch [2 (8.33\%), and visually significant cataract [4 (16.67\%)] were the complications observed in post-operative period.

Conclusion Primary Ahmed valve implantation may provide a safe and effective option in the management of childhood glaucoma associated with SWS.
\end{abstract}

\section{Introduction}

Glaucoma is one of the major causes of ocular morbidity in patients with Sturge-Weber syndrome (SWS). It presents in $30-70 \%$ of patients with SWS [1]. With the involvement of port wine stain over the eyelid its incidence increases. It is mostly unilateral but can present as bilateral also. The malformation of anterior chamber angle and increased episcleral venous pressure are the major mechanisms for glaucoma which have been demonstrated in the previous literature $[2,3]$.

Vaibhav Kumar Jain

vaibhav.jainaiims@gmail.com

1 Department of Ophthalmology, Army Hospital (Research and Referral), Delhi Cantt, New Delhi 110010, India

2 Department of Ophthalmology, Armed Forces Medical College, Pune, India

3 Department of Ophthalmology, Sanjay Gandhi Post Graduate Institute of Medical Sciences, Lucknow 226014 Uttar Pradesh, India
The management of childhood glaucoma associated with SWS has always been a challenging task to the clinician due to the complex multifactorial pathogenesis. Olsen et al. reported the success rate of $66.7 \%$ after one or more goniotomy or trabeculotomy in 16 eyes of glaucoma with SWS with a median follow-up of 5.4 years [4]. However, two-third of patients with initial gonitomy and half of patients with initial trabeculotomy required a second procedure [4]. In a series of 7 eyes, trabeculectomy was reported to be successful in 2 eyes without anti-glaucoma medications and 4 with mild medications in a follow-up that ranged from 9 months to 9 years [5]. Trabeculectomy has been associated with choroidal effusion in $24 \%$ of cases in a series by Iwasch et al. [6]. Mandal et al. in their series of 10 eyes of 9 patients reported a good control of IOP $(<16 \mathrm{~mm}$ $\mathrm{Hg}$ ) with only one eye developing choroidal effusion in the mean follow-up of 27.6 months after combined trabeculotomy-trabeculectomy [7]. Currently, there is no general consensus regarding the ideal procedure for childhood glaucoma associated with SWS.

Hypotony, serous, or hemorrhagic choroidal detachment, retinal detachment, and bleb failure are the real complications associated with glaucoma incisional procedures. 
Aqueous drainage implantation offers a good choice to control IOP due to relatively lesser complications associated with it [8]. Ahmed Implant is a valvular aqueous drainage implant which offers unidirectional flow to prevent postoperative hypotony which also does not require internal occlusion or external ligature as in non-valvular implants. The studies evaluating Ahmed valve implantation as a primary procedure in the childhood glaucoma associated with SWS have been few $[9,10]$. Herein, we report the efficacy and postoperative complications in managing these complicated cases by Ahmed valve implantation in 24 eyes which is a highest single center-based study.

\section{Materials and methods}

In this study, 24 eyes of 18 patients with childhood glaucoma associated with SWS who underwent Ahmed valve implantation were analyzed from 2008 to 2012 retrospectively. The ethical committee clearance was obtained for the study. The study was performed as per Declaration of Helinski. This study was performed in a tertiary eye care hospital.

This study included the cases of SWS which were clinically diagnosed by multidisciplinary approach involving neurology, pediatrics, and dermatology presented with the ophthalmic complaints. The ophthalmic examination included slit lamp examination wherever possible, IOP measurement by Perkin's Applanation tonometer. Corneal diameter, axial length, and fundus examination by $+90 \mathrm{D}$ lens and indirect ophthalmoscopy were also performed. Visual fields assessment, Ultrasonography B scan for choroidal thickening or hamartoma, was also done wherever possible. Gonioscopy was conducted in all patients to detect iridocorneal angle malformation. IOP measurement, optic nerve evaluation, as well as visual field testing represent the main procedures to diagnose and monitor glaucoma progression. The childhood glaucoma was diagnosed on the basis of high IOP, enlarged corneal diameter with/without optic disc cupping $>0.4$ or asymmetry of cup between two eyes. The clinical manifestations of SWS (age under 15 years) included port wine hamartomas on face, leptomeningeal angiomatosis, soft tissues, and bone hypertrophy, seizures, neurodevelopemental delay, and ophthalmic conditions such as glaucoma and choroidal hemangioma. The slit lamp examination was performed to look for conjunctival and episcleral venous dysplasia and tissue hypertrophy which have been considered as malformations traditionally [11]. After preoperative work-up of patients Ahmed valve (Ahmed Glaucoma Valves (Models: FP8 (96 $\mathrm{mm}^{2}$ ); FP7 (184 $\mathrm{mm}^{2}$ ), New World Medical Inc, Rancho Cucamonga, California, USA) surgery was planned as a primary procedure. All surgeries were conducted by single surgeon (JKSP) in a tertiary hospital after taking consent in general anesthesia. The surgical technique involved the creation of superotemporal conjunctival flap with superotemporal valve plate placement around $8 \mathrm{~mm}$ away from limbus and tube covered with $4 \times 4 \mathrm{~mm}$ partial thickness scleral flap taking consideration of scleral thinning. The placement of valve tube inside the anterior chamber was performed in such manner to prevent touching of anterior lens capsule and cornea. To prevent postoperative hypotony, partial ligation of tube with 7'o vicryl suture was performed and injection of sodium hyaluronate $1.4 \%$ was given into the anterior chamber. Postoperatively, patients received topical moxifloxacin $(0.5 \%)$, and prednisolone acetate $(1 \%)$ four times per day tapered over 3 weeks. Patients were followed-up on day 1, 1 week, 4 weeks, 3 months, and then quarterly.

The success was defined as $\mathrm{IOP}<21$ and $>5 \mathrm{~mm} \mathrm{Hg}$ without additional surgical maneuver with or without antiglaucoma medicines at 2 years of follow-up. Failure was defined as IOP $<5$ and $>21$ with anti-glaucoma medications or with additional surgical interventions. Statistical analysis was performed by SPSS software. The success rate (\%) and the mean \pm standard deviations (SD) for various preoperative variables were calculated. Paired $t$ ' test was used to compare quantitative variable (mean IOP, mean number of anti-glaucoma medications) between preoperative and postoperative at last follow-up. Patients were also assessed for intraoperative and postoperative complications.

\section{Results}

Twenty-four eyes of 18 patients of SWS with glaucoma were included. The various preoperative characteristics were shown in Table 1. All patients had Port wine Stain

Table 1 Various preoperative characteristics of children who underwent Ahmed valve implantation in glaucoma associated with Sturge-Weber syndrome

\begin{tabular}{ll}
\hline Characteristics & Values \\
\hline Mean age $( \pm \mathrm{SD})$ years & $\begin{array}{l}7.91 \pm 5.02 \\
(\mathrm{r}: 1-15)\end{array}$ \\
Sex ratio (male/female) & $16 / 8$ \\
Unilateral/bilateral & $18 / 3$ \\
Mean IOP at presentation $( \pm \mathrm{SD}) \mathrm{mm} \mathrm{Hg}$ & $34.04 \pm 4.97$ \\
& $(\mathrm{r}: 26-46)$ \\
Mean IOP on maximal anti-glaucoma & $26.21 \pm 3.52$ \\
medications $( \pm \mathrm{SD})$ mm Hg & $(\mathrm{r}: 22-36)$ \\
Preoperative mean number of anti-glaucoma & $3.92 \pm 0.28$ \\
drugs $( \pm \mathrm{SD})$ & \\
Mean axial length $( \pm \mathrm{SD}) \mathrm{mm}$ & $25.21 \pm 3.41$ \\
\hline
\end{tabular}

$S D$ standard deviation, IOP intraocular pressure, $r$ range 


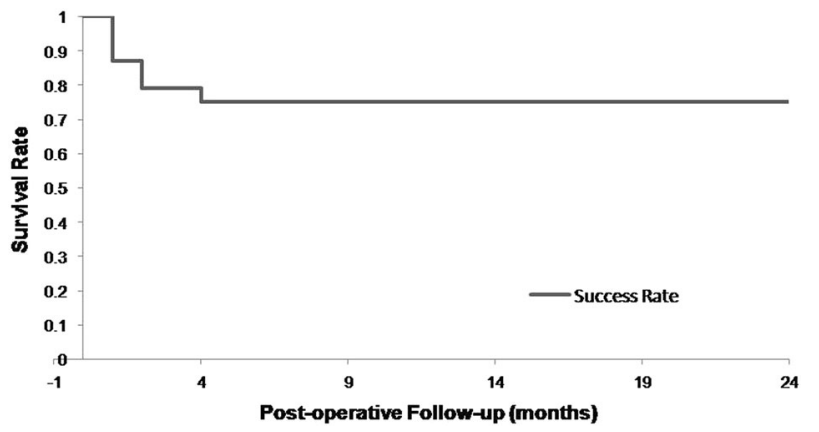

Fig. 1 Kaplan-Meier survival curve analysis for success rate of Ahmed valve in childhood glaucoma associated with Sturge-Weber syndrome

involving eyelids, on facial skin. Bilateral cutaneous lesions were seen in three $(16.67 \%)$ of our patients. Other ocular abnormalities encountered was dilated episcleral vessels in all cases and choroidal hemangioma in four eyes (16.67\%). For all patients, gonioscopy, revealed no angle anomalies.

The mean IOP at first clinical presentation was $34.04 \pm$ 4.97 (range: $26-46$ ) $\mathrm{mm} \mathrm{Hg}$ whereas mean IOP on maximal anti-glaucoma medications (beta blocker, prostaglandin, and carbonic anhydrase inhibitor) was $26.21 \pm 3.52$ (range: 22-36). After Ahmed valve implantation the mean IOP was significantly reduced $(12.27 \pm 1.27 \mathrm{~mm} \mathrm{Hg})$ at 2 years of follow-up $(P=0.001)$. A statistically significant reduction in mean number of anti-glaucoma medications was also observed $(3.92 \pm 0.28$ to $1.5 \pm 1 ; P=0.015)$. The success rate by Kaplan-Meier survival curve analysis was $75 \%$ (Confidence Interval 95\%, 60 to 100\%) at the mean followup of $2.12 \pm 0.87$ years (Fig. 1).

The FP8 model of Ahmed valve was used most commonly (18 eyes) followed by normal adult model FP7 (6 eyes) which was performed in older children ( $>12$ years having axial length more than $22 \mathrm{~mm}$ ).

In three eyes, lens aspiration with intraocular lens implantation was also performed due to the presence of visually significant cataract at the time of Ahmed valve implantation. Apparently, lens aspiration did not seem to affect the results postoperatively as all cases were uneventful with controlled IOP. Except one eye having amblyopia, all others had visual acuity of $6 / 9$ or more at 2 years of follow-up.

The various post-operative complications are shown in Table 2. Three (12.5\%) eyes experienced very low IOP $(<5$ $\mathrm{mm} \mathrm{Hg}$ ) with recurrent shallow anterior chamber in the initial postoperative period for which anterior chamber reformation was performed with sodium hyaluronate thrice, therefore they were labeled as failed according to the failure criteria defined in methods. However, hypotony resolved with the formation of anterior chamber within 2 weeks of its first appearance. Postoperative hyphema was observed in four eyes of four cases which resolved within a week. Three
Table 2 Various complications after Ahmed valve surgery in childhood glaucoma associated with Sturge-Weber syndrome

\begin{tabular}{ll}
\hline Complications & $\begin{array}{l}\text { Number of } \\
\text { eyes }(\%)\end{array}$ \\
\hline Hypotony & $3(12.5)$ \\
Choroidal detachment & $3(12.5)$ \\
Hyphema & $4(16.67)$ \\
Tube-cornea touch & $2(8.33)$ \\
$\begin{array}{l}\text { Visually significant cataract } \\
\text { Failure rate (intraocular pressure }<5 \text { and }>21 \text { with } \\
\text { anti-glaucoma medications or with additional } \\
\text { surgical interventions) }\end{array}$ & $4(16.67)$ \\
\hline
\end{tabular}

eyes had serous choroidal detachment with shallow anterior chamber which was treated conservatively with systemic steroids and resolved with IOP control in 2 weeks. Tubecornea touch was present in two eyes which required reinsertion of tube therefore also labeled as failed cases according to the criteria of failure. One eye experienced raised IOP $(>21 \mathrm{~mm} \mathrm{Hg})$ on maximal anti-glaucoma medicines for which child underwent additional Ahmed valve implantation. Four eyes having very minimal lenticular opacity not obscuring the visual axis before surgery, developed visually significant cataract during the follow-up period for which the child underwent lens aspiration with intraocular lens implantation. Overall 7 (29.16\%) eyes had one or more complications after Ahmed valve implantation. The visual acuity of $6 / 9$ or more on Snellen chart was observed in six eyes with one or more complications whereas visual acuity of 6/24 vision was observed in one eye due to amblyopia.

\section{Discussion}

Managing glaucoma in SWS is a challenge due to poor control with medical management and surgical management remains the mainstay in treating these cases. Various factors such as large corneal diameter, large axial length, scleral thinning, angle anomalies, raised episcleral venous pressure make the surgical procedure challenging and the outcome is unpredictable with conventional anti-glaucoma surgeries. Aqueous drainage implant was found to be associated with greater control of IOP compared to trabeculectomy with mitomycin-C in the first 2 years of life in a retrospective comparative study of 46 eyes of 32 children aged 2 years or younger [12]. Hence, Ahmed valve has been considered as a primary procedure.

Hamush et al. in their retrospective series of 11 eyes implanted with Ahmed valve in childhood glaucoma associated with SWS reported the cumulative probability of success rate being $79 \%$ at 2 years of follow-up which came 
down to $30 \%$ after 5 years in 11 eyes [10]. Ahmed valve was also found to be an effective and safe procedure in a single case report of SWS at 1 year of follow-up [8]. We also reported significant reduction of IOP and mean number of anti-glaucoma medications in our patients following implantation of Ahmed valve. Our success rate was 75\% which was comparable to the case series by Hamush et al. [10]. However we had more number of eyes compared to their series.

In a major review of Ahmed valve in pediatric glaucoma by Nassiri et al. the success rate of Ahmed valve was better than that with trabeculectomy with MMC in previous studies [12-14] and this was found to be an effective procedure for control of IOP that can last for 5 or more years [15]. However, it may be associated with pupillary irregularity, lens opacification, and tube related complications particularly in the first year of life as the globe enlarges with age.

Hypotony is the major concern after surgery particularly in SWS. With glaucoma implants there is a risk of hypotony with further devastating complications like choroidal hemorrhage. Therefore, we partially ligated the tube of Ahmed valve to prevent hypotony. This method has also been applied successfully by Chen et al. in their series of refractory pediatric glaucoma [16]. This may avoid the chances of hypotony in the postoperative period. We reported hypotony in three $(12.5 \%)$ eyes with shallow anterior chamber which required reformation. The reported incidence of hypotony in Ahmed implant in childhood glaucoma ranged from 11 to $25 \%$ in a review by Nassiri et al. [15].

Tube-cornea touch is also a worrisome complication in pediatric aqueous drainage implant surgery as it requires the revision of surgery and further expose the child to the risk of general anesthesia. We reported tube-cornea touch in two eyes $(8.33 \%)$ for which tube reinsertion was done again. However, it has been reported as $18.5 \%$ after Ahmed implant surgery in childhood refractory glaucoma in an earlier retrospective series [17].

Although our study is limited by short follow-up, this is a highest single center-based study that shares a useful experience in managing such cases and their complications which has not been reported till now. We had more success rate $(75 \%$ versus $66.7 \%)$ compared to the earlier series having angle surgeries which frequently required repeat surgeries further exposing the child to the risk of general anesthesia whereas eye requiring second surgical intervention was labeled as failed in our study [4]. Further, potentially vision threatening complication like choroidal effusion (12.5\% versus $24 \%$ ) was less commonly encountered compared to the earlier study involving trabeculectomy in pediatric glaucoma [6]. In view of our study findings the primary Ahmed valve implantation may provide a safe and effective option in the management of childhood glaucoma associated with SWS especially in the absence of angle anomalies.

\section{Summary}

\section{What was known before}

- Very few study on the use of Ahmed valve in management of glaucoma associated with Sturge-Weber syndrome.

\section{What this study adds}

- This is a highest single center-based study that shares a useful experience in managing such cases and their complications which has not been reported till now.

\section{Compliance with ethical standards}

Conflict of interest The authors declare that they have no conflict of interest.

\section{References}

1. Sullivan TJ, Clarke MP, Morin JD. The ocular manifestations of the Sturge-Weber syndrome. J Pediatr Ophthalmol Strabis. 1992;29:349-56.

2. Weiss DI. Dual origin of glaucoma in encephalotrigeminal hemangiomatosis. Trans Ophthalmol Soc. 1973;93:477-93.

3. Cibis GW, Tripathi RC, Tripathi BJ. Glaucoma in Sturge-Weber syndrome. Ophthalmology. 1984;91:1067-71.

4. Olsen KE, Huang AS, Wright MM. The efficacy of goniotomy/ trabeculotomy in early-onset glaucoma associated with the SturgeWeber syndrome. J AAPOS. 1998;2:365-8.

5. Ali MA, Fahmy IA, Spaeth GL. Trabeculectomy for glaucoma associated with Sturge-Weber syndrome. Ophthalmic Surg. 1990;21:352-5.

6. Iwach AG, Hoskins HD Jr, Hetherington J Jr, Shaffer RN. Analysis of surgical and medical management of glaucoma in SturgeWeber syndrome. Ophthalmology. 1990;97:904-9.

7. Mandal AK. Primary combined trabeculotomy-trabeculectomy for early onset glaucoma in Sturge-Weber syndrome. Ophthalmology. 1999;106:1621-7.

8. Gedde SJ, Schiffman JC, Feuer WJ, Herndon LW, Brandt JD, Budenz DL. Treatment outcomes in the Tube Versus Trabeculectomy (TVT) study after five years of follow-up. Am J Ophthalmol. 2012;153:789-803.

9. Wu SC, Lin KK. Ahmed glaucoma valve implant for childhood glaucoma in Sturge-Weber syndrome with choroidal hemangioma. Chang Gung Med J. 2006;29:528-31.

10. Hamush NG, Coleman AL, Wilson MR. Ahmed glaucoma valve implant for management of glaucoma in Sturge-Weber syndrome. Am J Ophthalmol. 1999;128:758-60.

11. Parsa CF. Focal venous hypertension as a pathophysiologic mechanism for tissue hypertrophy, port wine stains, the Sturge 
Weber syndrome, and related disorders: proof of concept with novel hypothesis for underlying etiological cause. Trans Am Ophthalmol Soc. 2013;111:180-215.

12. Beck AD, Freedman S, Kammer J, Jin J. Aqueous shunt devices compared with trabeculectomy with Mitomycin-C for children in the first two years of life. Am J Ophthalmol. 2003;136:994-1000.

13. Pakravan M, Nikkhah H, Yazdani S, Baradaran Rafiei AR. Trabeculectomy with mitomycin $\mathrm{C}$ versus Ahmed glaucoma implant with mitomycin $\mathrm{C}$ for treatment of pediatric aphakic glaucoma. $\mathrm{J}$ Glaucoma. 2007;16:631-6.
14. Netland PA, Walton DS. Glaucoma drainage implants in pediatric patients. Ophthamic Surg. 1993;24:723-9.

15. Nassiri N, Nour-Mahdavi K, Colemann AL. Ahmed glaucoma valve in children: a review. Saudi J Ophthalmol. 2011;25:317-27.

16. Chen TC, Bhatia LS, Walton DS. Ahmed valve surgery for refractory pediatric glaucoma: a report of 52 eyes. J Pediatr Ophthalmol. 2005;42:274-83.

17. Englert JA, Freedman SF, Cox TA. The ahmed valve in refractory glaucoma. Am J Ophthalmol. 1999;127:34-42. 